\title{
Successful endoscopic treatment of an appendicocutaneous fistula using endoloops
}

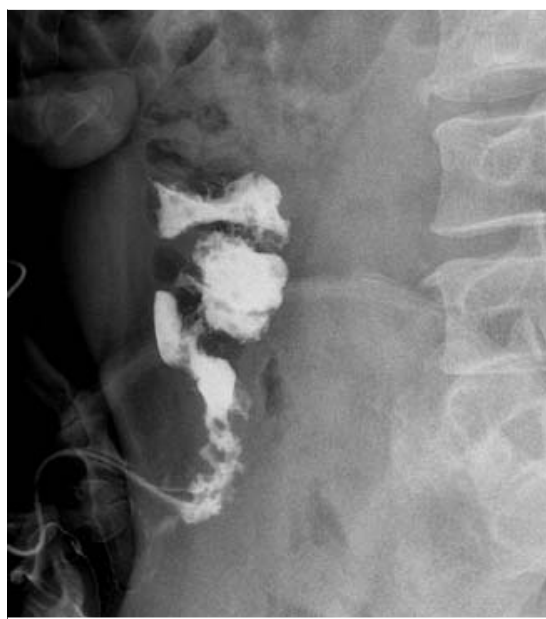

- Fig. 1 Abdominal wall radiograph showing the abdominal wall fistula connected with the ileocecal region.

An appendicocutaneous fistula is a rare complication of appendectomy [1]. Moreover, the fistula can persist [2]. A 52-year-old man presented to our department with a 3-month history of purulent abdominal wall sinus that developed after appendectomy 3 months ago. Abdominal wall radiography revealed an abdominal wall fistula connecting with the ileocecal region (> Fig.1). Under colonoscopy, we identified the swollen and purulent appendix stump with black stitching residue ( $>$ Video $\mathbf{1}$ ). After repeated washing of the appendix stump ( $\triangleright$ Video 1 ), milky pus remained around the stitching residue ( $>$ Fig. 2 ). Using grasping forceps, we easily removed the stitching residue ( $\downarrow$ Video 1$)$. Saline solution with methylene blue was injected from the ostium of the abdominal wall fistula, and the inlet of the fistula was detected at the appendix stump ( $\mathbf{F i g . 3}$ ). Normal saline was then injected repeatedly from the abdominal outlet of the sinus to wash the fistula ( $\vee$ Video 1 ). After washing, we released a nylon ring into the ileocecal region ( $\triangleright$ Video $\mathbf{1}$ ). Eight clips were used to fasten the nylon ring around the inlet of the fistula at the appendix stump ( $\triangleright$ Video 1 ). We then tightened
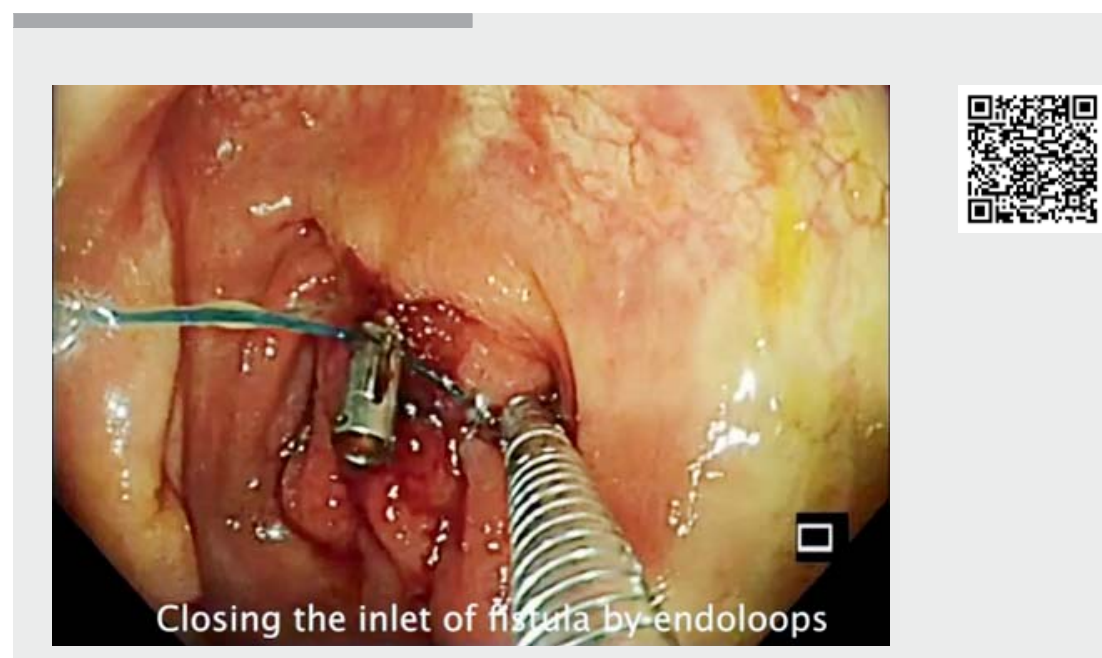

Video 1 Appendicocutaneous fistula treated using endoscopic endoloops.

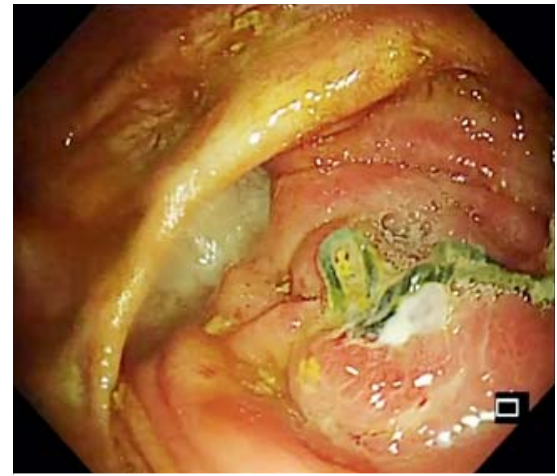

Fig. 2 Milky pus around the stitching residue.

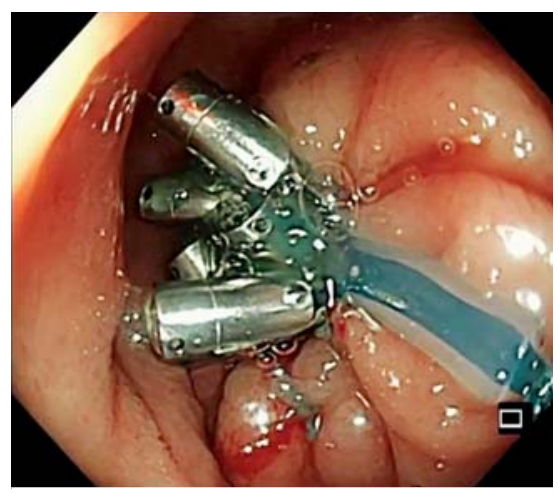

Fig. 4 Closing of the fistula inlet using endoloops.

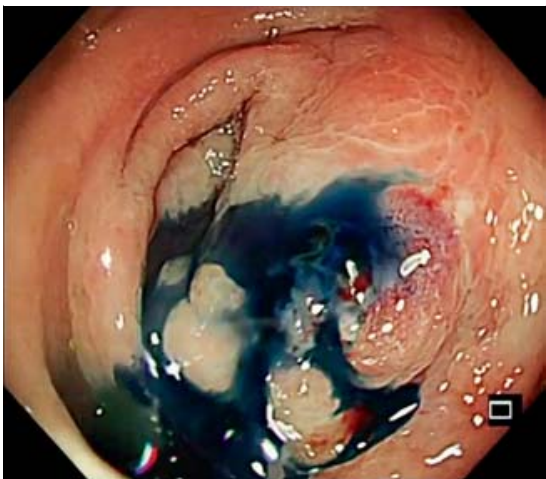

Fig. 3 Inlet of the fistula at the appendix stump.

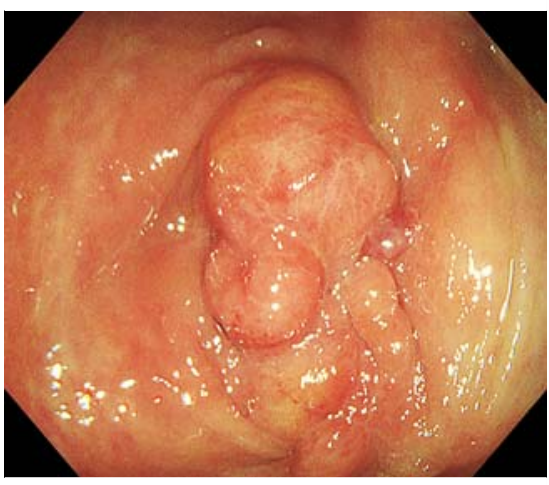

- Fig. 5 Inlet of the fistula healing 2 weeks after treatment. 
and released the nylon ring ( $\triangleright$ Fig.4). Methylene blue dye was again injected from the abdominal wall sinus outlet ( $\triangleright$ Video 1); the dye was refluxed back, and the ileocecal region did not show methylene blue. We found two outlets of the fistula ( Video 1). Two drainage tubes were placed at the outlets ( Video 1 ). Two weeks later, we noted that the inlet and the outlets of the fistula were healing ( Fig.5). The use of endoscopic endoloops is a new strategy to promote healing of an appendicocutaneous fistula without surgery.

Endoscopy_UCTN_Code_CPL_1AJ_2AG

\section{Acknowledgments}

This article was supported by the Projects of the Bureau of Science and Technology, Nanchong City (grant nos. 18SXHZ0109, 18SXHZ0466).

\section{Competing interests}

The authors declare that they have no conflict of interest.
The authors

Zhang Tao ${ }^{1}$, Dongbing Zhou ${ }^{2}$, Xiaosan $\mathrm{Hu}^{1}$, Mingyang Ren ${ }^{2}$, Yiwen $\mathrm{Yu}^{1}$, Xiaoqing Zhou ${ }^{1}$

1 Department of Gastroenterology, Nanchong Central Hospital, Nanchong City, Sichuan, China

2 Department of Gastrointestinal Surgery, Nanchong Central Hospital, Nanchong City, Sichuan, China

Corresponding author

\section{Xiaoqing Zhou, MD}

Department of Gastroenterology, Nanchong Central Hospital, Nanchong City, Sichuan, 637000, China

Fax: +86-10-67788052

zhangtao195932@163.com

\section{References}

[1] Brünagel G, Decker P, Hirner A. Delayed appendico-cutaneous fistula - a rare complication of simple abdominal drainage. Zentralbl Chir 1996; 121: 67-69

[2] Shamim M, Haider SA, Iqbal SA. Persistent appendiceal faecal fistula following a complicated open appendicectomy. J Pak Med Assoc 2009; 59: 181-183
Bibliography

DOI https://doi.org/10.1055/a-1134-4630

Published online: 15.4.2020

Endoscopy 2020; 52: E383-E384

(c) Georg Thieme Verlag KG

Stuttgart · New York

ISSN 0013-726X

\section{ENDOSCOPY E-VIDEOS}

https://eref.thieme.de/e-videos

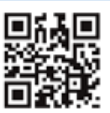

Endoscopy E-Videos is a free access online section, reporting on interesting cases and new

techniques in gastroenterological endoscopy. All papers include a high quality video and all contributions are freely accessible online.

This section has its own submission website at https://mc.manuscriptcentral.com/e-videos 